Epidemiology and Infection

cambridge.org/hyg

\section{Original Paper}

Cite this article: Kimura T, Stanhope S, Sugitani T (2020). Excess length of hospital stay, mortality and cost attributable to Clostridioides (Clostridium) difficile infection and recurrence: a nationwide analysis in Japan. Epidemiology and Infection 148, e65, 1-11. https://doi.org/10.1017/ S0950268820000606

Received: 24 June 2019

Revised: 19 December 2019

Accepted: 26 February 2020

Key words:

Clostridium difficile; excess length of hospital stay; immortal time; Japan; mortality

Author for correspondence:

T. Kimura, E-mail: tomomi.kimura@astellas. com

\title{
Excess length of hospital stay, mortality and cost attributable to Clostridioides (Clostridium) difficile infection and recurrence: a nationwide analysis in Japan
}

\author{
T. Kimura ${ }^{1}$ (D) S. Stanhope ${ }^{2}$ and T. Sugitani ${ }^{1}$
}

${ }^{1}$ Astellas Pharma Inc., Tokyo, Japan and ${ }^{2}$ Astellas Pharma US LLC, Northbrook, IL, USA

\begin{abstract}
Clostridioides (Clostridium) difficile infection (CDI) is the leading cause of infectious diarrhoea in hospitalised patients, representing a substantial economic burden driven mainly by increased length of hospital stay (LoS). Currently in Japan, limited evidence on CDI-associated excess LoS is available. We conducted a retrospective, matched-cohort study using a large, Japanese, hospital-based administrative database. CDI was defined as CDI treatment plus either CDI diagnosis or positive enzyme immunoassay result. Propensity score matching at the time of $\mathrm{CDI}$ or recurrent $\mathrm{CDI}$ (rCDI) onset was applied to adjust baseline confounding and immortal time bias. The analysis included 5994054 hospitalisation records during 20082017, of which 11823 were identified as CDI and 1359 as rCDI. The median excess LoS attributable to CDI and rCDI was 3 days and 6.5 days, respectively. The excess mortality attributable to CDI was $6.9 \%$; there was no excess mortality attributable to rCDI $(-1.9 \%)$. The median difference in costs attributable to CDI and rCDI during the residual stay was JPY 130296 (USD 1185) and JPY 81054 (USD 737) per hospitalisation, respectively. By adjusting the biases, the burden of CDI in Japan was evaluated. The findings could support decision making and resource allocation for CDI management in Japanese hospitals.
\end{abstract}

\section{Introduction}

Clostridioides (Clostridium) difficile infection (CDI) is the leading cause of infectious diarrhoea in hospitalised patients, and $\sim 10-33 \%$ of CDI cases result in recurrent infection (rCDI) [1-3]. It is generally accepted that CDI prolongs length of hospital stay (LoS), which drives the economic burden of nosocomial CDI [4-11]. A recent systematic review of studies in Japan [12] reported that there are limited data on CDI-associated LoS and costs $[2,4,13]$, and further studies are therefore needed to support decision-making and resource allocation in the management of CDI in Japan.

When assessing the impact of CDI on excess LoS or mortality, particular attention is needed for immortal time bias because nosocomial CDI occurs after the admission (i.e. timedependent exposure). Without controlling for immortal time bias, the excess LoS will be overestimated $[6-8,10,14-18]$. Also, it is very likely that patients with CDI have different baseline characteristics from those without CDI, which can be the confounding factors.

To manage these biases, we conducted a retrospective, propensity score matched-cohort study using a large, Japanese, hospital-based administrative database to evaluate excess LoS, mortality and costs attributable to CDI or rCDI.

(c) The Author(s), 2020. Published by Cambridge University Press. This is an Open Access article, distributed under the terms of the Creative Commons AttributionNonCommercial-NoDerivatives licence (http:// creativecommons.org/licenses/by-nc-nd/4.0/), which permits non-commercial re-use, distribution, and reproduction in any medium, provided the original work is unaltered and is properly cited. The written permission of Cambridge University Press must be obtained for commercial re-use or in order to create a derivative work.

\section{CAMBRIDGE} UNIVERSITY PRESS

\section{Methods}

This was a retrospective, matched-cohort study of hospitalised patients in Japan.

\section{Data source}

A hospital-based administrative database provided by Medical Data Vision was used as a data source [19]. The dataset covered more than 19 million outpatients and inpatients who visited 320 Diagnostic Procedure Combination (DPC) hospitals in Japan as of November 2017 (around 19\% of 1666 DPC hospitals in 2016). DPC is a flat-fee payment system for inpatients, in which around $20 \%$ of hospitals in Japan participate. This dataset includes diagnosis, laboratory results (limited, see below) and transaction-level records of prescriptions and procedures, even during hospitalisation. The maximum age at admission was rounded at 90 years in this database to increase patient anonymity. 


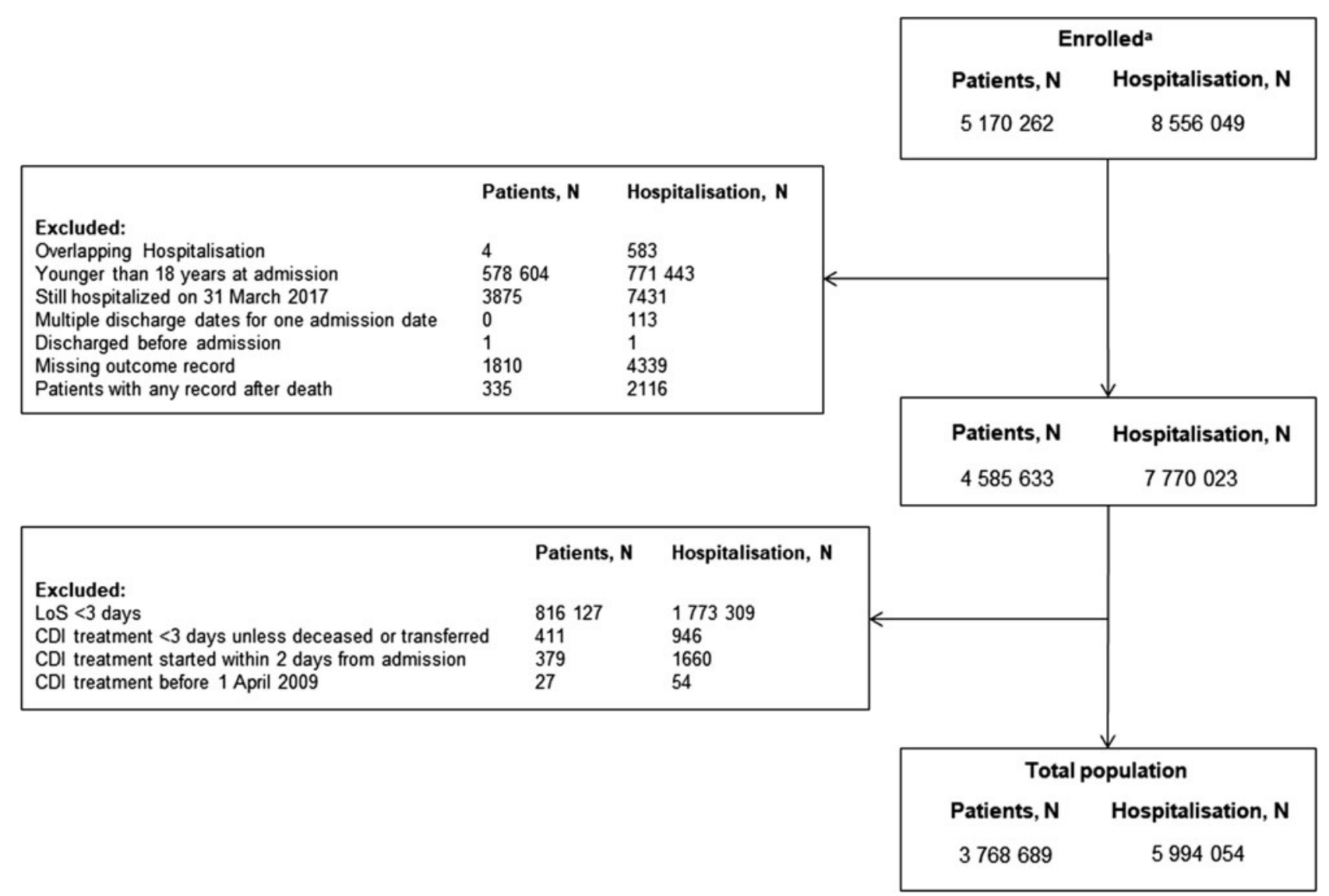

Fig. 1. Patients and hospitalisations. ${ }^{\mathrm{a}} \mathrm{CDI}$-associated hospitalisation records with admission date on or after 1 April 2008 and discharge date on or before $31 \mathrm{March}$ 2017: CDI-associated hospitalisation was defined as a hospitalisation with CDI treatment plus either diagnosis or laboratory test positive results. LoS, length of hospital stay; CDI, Clostridioides (Clostridium) difficile infection.

The study was approved by the Astellas Medical Affairs Japan Protocol Review Committee on 19 October 2017. The study was conducted following Good Pharmacoepidemiology Practice [20].

\section{Study population}

All hospitalisation records with admission date on or after 1 April 2008 and discharge date on or before 31 March 2017 were extracted. Hospitalisations were excluded from the analysis if the patient was aged $<18$ years at admission, the LoS was $<3$ days or the outcome records were missing (e.g. death).

CDI-associated hospitalisation was defined as a hospitalisation with CDI treatment plus either CDI diagnosis or positive enzyme immunoassay (EIA) test result, with the time of CDI onset defined as the start of treatment.

CDI treatment was defined as prescription of oral or intravenous (IV) metronidazole (MNZ) or oral vancomycin (VCM), initiated $>2$ days after admission to hospital (as an indicator of hospital-onset CDI) and $\geq 56$ days after the end date of any previous CDI treatment. Medications were defined using the first 7 digits of YJ code (Japanese drug codes): 6419002 for oral MNZ, 6419401 for IV MNZ and 6113001 for oral VCM. CDI treatment must have been continued for $\geq 3$ days unless the patient was discharged, transferred or deceased. A treatment episode could have an interval of 1-2 days without CDI medication, i.e. the next prescription for CDI treatment within 2 days was considered as a continuous treatment.

CDI diagnosis was defined as an International Classification of Diseases 10th revision (ICD-10) code of A04.7 ('Enterocolitis due to Clostridium difficile'), in the same month as the CDI treatment initiation and without suspicion flag.

The results from EIA for $C$. difficile toxin A/B +/- glutamate dehydrogenase $(\mathrm{GDH})$ antigen were obtained separately from nine out of 320 hospitals via Medical Data Vision. Positive results for the EIA can be positive for toxin A, toxin B and/or GDH, so were indistinguishable. Positive results obtained \pm 1 day from the start of the CDI treatment were considered part of the CDI definition.

rCDI-associated hospitalisation was defined as re-initiation of CDI treatment during the period between the end of prior CDI treatment plus 3 days and within 56 days ( 8 weeks) from the initiation of the previous CDI treatment, during the same hospital stay.

The total cohort included non-CDI and CDI hospitalisations that fulfilled the inclusion criteria and did not meet the exclusion criteria. The CDI hospitalisations were divided into rCDI and non-rCDI hospitalisations. CDI and rCDI onset date was defined as the date of CDI treatment initiation or re-initiation. Time to CDI was calculated as the number of days between admission and CDI onset. Time to rCDI was estimated as days between the end of initial treatment and rCDI onset. The Charlson comorbidity index was estimated using an algorithm reported by Quan et al. [21, 22]. 
Table 1. Demographic and clinical characteristics in the total CDI cohort and in the CDI-matched cohort

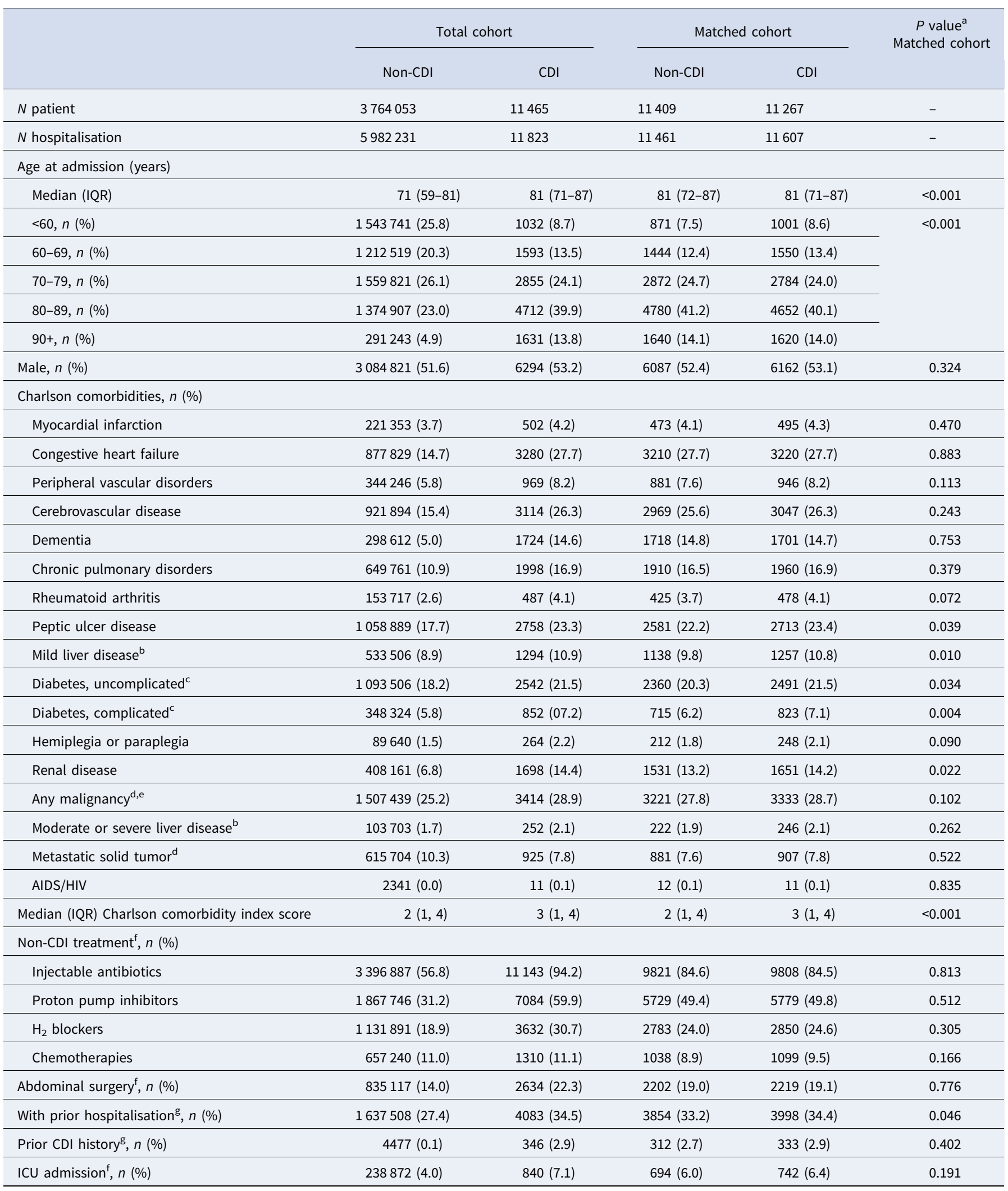

CDI, Clostridioides (Clostridium) difficile infection; IQR, interquartile range; ICU, intensive care unit.

avariables and $\chi^{2}$ tests for binary or categorical variables.

Varidables and $\chi^{2}$ tests
Mutually exclusive.

eIncluding lymphoma and leukemia except malignant neoplasm of skin.

During the stay.

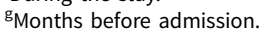


Table 2. Demographic and clinical characteristics in the total rCDI cohort and in the rCDI matched cohort

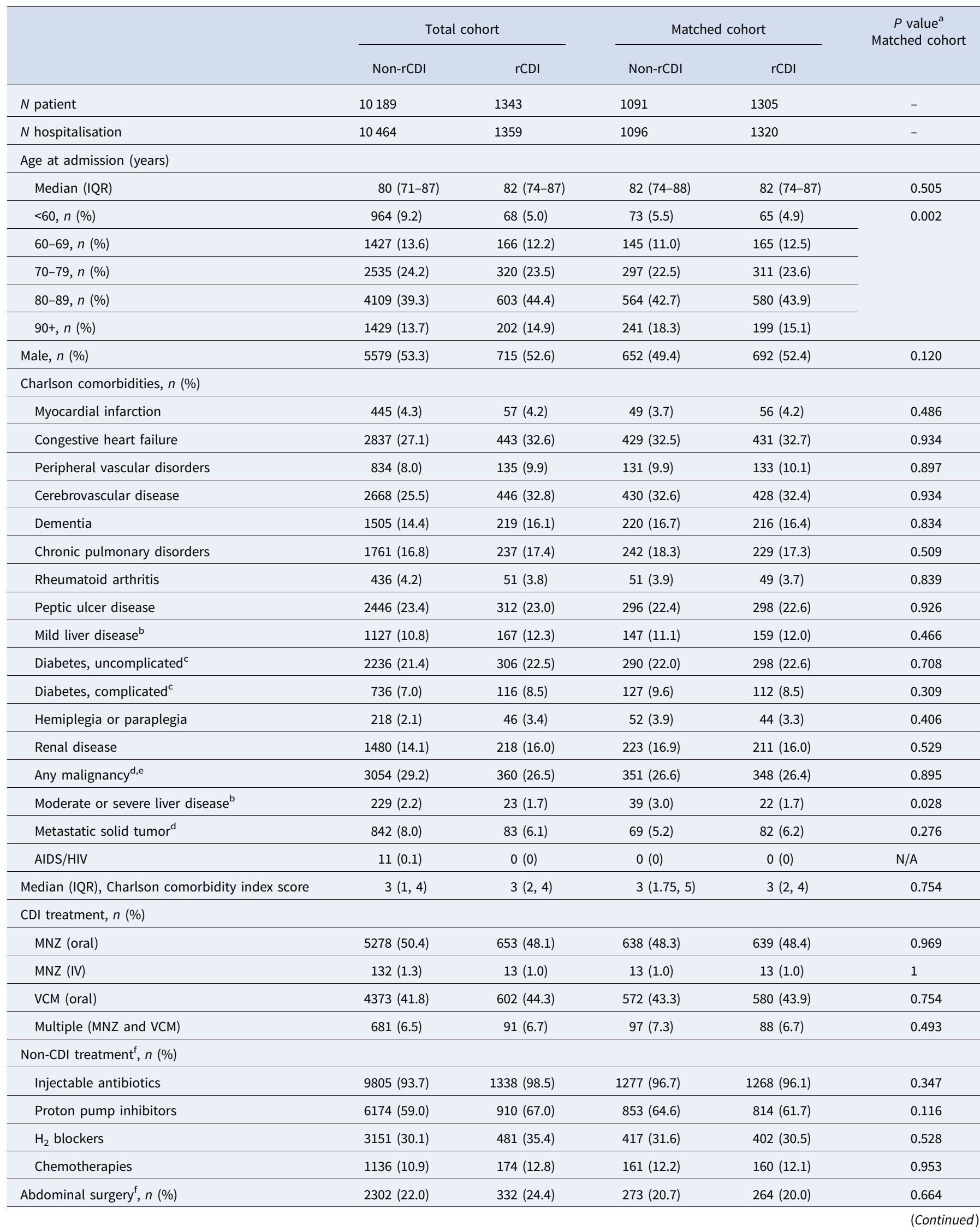


Table 2. (Continued.)

\begin{tabular}{|c|c|c|c|c|c|}
\hline & \multicolumn{2}{|c|}{ Total cohort } & \multicolumn{2}{|c|}{ Matched cohort } & \multirow{2}{*}{$\begin{array}{c}P \text { value }^{\mathrm{a}} \\
\text { Matched cohort }\end{array}$} \\
\hline & Non-rCDI & rCDI & Non-rCDI & rCDI & \\
\hline With prior hospitalisation ${ }^{\mathrm{g}}, n(\%)$ & $3653(34.9)$ & $430(31.6)$ & $418(31.7)$ & $420(31.8)$ & 0.933 \\
\hline Prior CDI history ${ }^{\mathrm{g}}, n(\%)$ & $303(2.9)$ & $43(3.2)$ & $37(2.8)$ & $41(3.1)$ & 0.646 \\
\hline ICU admission ${ }^{f}, n(\%)$ & $738(7.1)$ & $102(7.5)$ & $107(8.1)$ & $95(7.2)$ & 0.380 \\
\hline
\end{tabular}

CDI, Clostridioides (Clostridium) difficile infection; rCDI, recurrent CDI; IQR, interquartile range; MNZ, metronidazole; IV, intravenous; ICU, intensive care unit.

${ }^{a}$ Variables and $\chi^{2}$ tests for binary or categorical variables.

b,c,d Mutually exclusive.

e Including lymphoma and leukemia except malignant neoplasm of skin.

fDuring the stay.

${ }^{g}$ Months before admission.

\section{Statistical analyses}

Analyses were performed using hospitalisation (not patient) as an analysis unit with the ' $R$ ' analysis package (version 3.3.2). The goal of our analysis was to quantify the effects of an in-hospital CDI (or rCDI) on LoS, mortality and hospitalisation costs. To minimise the effects of confounding and immortal time bias, time-dependent propensity score matching was performed [9]. One non-CDI hospitalisation was matched to one CDI hospitalisation from a different patient at the relative (with respect to the beginning of the stay) time of CDI onset using 1:1 greedy matching of logit-transformed CDI propensities (caliper $0.2 \times$ the standard deviation of the logit propensities), with replacement [23]. Similarly, one non-rCDI hospitalisation was matched to one rCDI hospitalisation at the time of rCDI onset.

Propensity scores were estimated for each hospitalisation using logistic regression with CDI (or rCDI) occurrence as a dependent variable, and the exploratory variables identified either: (a) at hospital admission (time-fixed covariates) or (b) during the period between admission and CDI onset or between the end of prior CDI treatment and rCDI onset (time-varying covariates). The time-fixed covariates included age at admission, sex, Charlson comorbidity index and its weighted score, calendar quarter of admission, CDI treatment for the initial episode (only for rCDI matching), hospitalisation history in the prior 6 months and CDI history in the prior 6 months. The time-varying covariates were the use of non-CDI injectable antibiotics, proton-pump inhibitor (PPI), $\mathrm{H}_{2}$ blockers, chemotherapy, abdominal surgery and intensive care unit (ICU) admission. The code list for medications and procedures is provided in Supplement 1.

\section{Excess length of hospital stay, mortality and cost}

LoS was estimated as days between admission and discharge, death or transfer to another hospital, whichever occurred first. The residual LoS was the days between matching date to discharge, death or transfer. The excess LoS was estimated as the median or mean of the differences in residual LoS between each matched pair. The discharge hazard ratio and 95\% confidence intervals (CI) were estimated using stratified Cox regression including matched pair as strata, and censoring transfer to another hospital. A sensitivity analysis was performed using a Kaplan-Meier (KM) method, with transfer to another hospital as a censoring event, while dissociating the matched pairs.

Excess hospital mortality (\%) and 95\% CI were estimated in the matched CDI/rCDI cohorts. The mortality hazard ratio and 95\% CI were estimated using stratified Cox regression including matched pair as strata, and censoring hospital discharge and transfer to another hospital.

Medical expenditure was estimated as the sum of all costs during the specified period. The excess costs were estimated as the median or mean of the differences in total medical expenditure during the residual stay between the matched pair. Costs were indicated in JPY and USD (costs in JPY were converted to USD at a rate of USD $1=$ JPY 110 ).

\section{Results}

\section{Study population}

Data relating to 8556049 hospitalisations for 5170262 patients were extracted from the database, and after applying the exclusion criteria, 5994054 hospitalisations remained in the analysis dataset (Fig. 1). Of these, 11823 (0.17\%) were CDI-associated hospitalisations, and of the CDI hospitalisations, 1359 (11.5\%) were identified as rCDI hospitalisations. CDI hospitalisations tend to occur in patients who were older, had slightly higher co-morbidity scores and had a higher prevalence of antibiotics, PPI and $\mathrm{H}_{2}$ blocker use, greater incidence of previous abdominal surgery and higher rates of prior CDI compared with non-CDI hospitalisations (Table 1). Patients with rCDI hospitalisations were slightly older than non-rCDI patients, with more antibiotic, PPI and $\mathrm{H}_{2}$ blocker prescriptions (Table 2).

A total of 11607 CDI hospitalisations were matched with 11 461 non-CDI hospitalisations $(1.8 \%$ of these were matched to $\geq 2$ different CDI hospitalisations at different onset timings) and 1320 rCDI hospitalisations were matched to 1096 non-rCDI hospitalisations. Matched cohort was well balanced for clinical characteristics (Tables 1 and 2).

\section{Excess length of hospital stay}

Before matching, the median difference in LoS between non-CDI hospitalisations and CDI hospitalisations was 40 days (interquartile range (IQR): 20,75$)$, whereas the median difference between the matched CDI-non-CDI pair was 3 days (IQR: -14, 22) (Table 3). Similarly, the median difference in LoS between non-rCDI hospitalisations and rCDI hospitalisations was 62 days (IQR: 44, 79.5) before matching, whereas the median difference between the matched rCDI-non-rCDI pair was 6.5 days (IQR: $-18,40)$.

In the KM analysis (censoring transfer to other hospital), the difference in median KM estimates between CDI hospitalisations 
Table 3. Summary of LoS outcomes in the total CDI/rCDI cohorts and the matched CDI/rCDI cohorts

\begin{tabular}{|c|c|c|c|c|c|c|c|c|}
\hline & \multicolumn{2}{|c|}{ Before matching } & \multicolumn{2}{|c|}{ After matching } & \multicolumn{2}{|c|}{ Before matching } & \multicolumn{2}{|c|}{ After matching } \\
\hline & Non-CDI & CDI & Non-CDI & CDI & Non-rCDI & $\mathrm{rCDI}$ & Non-rCDI & rCDI \\
\hline$N$ of unique hospitalisations, $n$ & 5982231 & 11823 & 11461 & 11607 & 10464 & 1359 & 1096 & 1320 \\
\hline$N$ of matched hospitalisations & - & - & 11607 & 11607 & - & - & 1320 & 1320 \\
\hline \multicolumn{9}{|l|}{ LoS, days } \\
\hline Median (IQR) & $11(6,22)$ & $51(26,97)$ & $47(23,88)$ & $51(25.5,95)$ & $46(24,87)$ & $108(68,166.5)$ & $90(59,155)$ & $106(67,163)$ \\
\hline Mean (S.D.) & $19.4(31.88)$ & $78.9(99.18)$ & $69.8(86.39)$ & $73.4(77.37)$ & $71.2(92.86)$ & $138.5(123.24)$ & $127.3(136.44)$ & $136.9(123.70)$ \\
\hline Range & 3-3031 & $3-2518$ & $3-1868$ & 3-1691 & $3-2518$ & $15-1725$ & $18-2086$ & $15-1725$ \\
\hline \multicolumn{9}{|c|}{ Days from matching date to discharge } \\
\hline Mean (S.D.) & - & & - & & - & & $55.2(77.97)$ & $69.6(92.30)$ \\
\hline \multirow[t]{2}{*}{ Range } & - & & - & & - & & $3-1526$ & $3-1386$ \\
\hline & Crude & & Adjusted & & Crude & & Adjusted & \\
\hline \multicolumn{9}{|l|}{ Excess LoS, days } \\
\hline Median (IQR) & $40(20,75)$ & & $3(-14,22)$ & & $62(44,79.5)$ & & $6.5(-18,40)$ & \\
\hline Mean $(95 \% \mathrm{Cl})$ & $59.5(57.8,61.3)$ & & $3.7(2.0,5.3)$ & & $67.3(60.5,74.0)$ & & $14.3(7.8,20.8)$ & \\
\hline $\begin{array}{l}\text { Difference in median KM } \\
\text { estimates, days }\end{array}$ & - & & $4(3,14)^{a}$ & & - & & $19(6,34)$ & \\
\hline Discharge hazard ratio $(95 \% \mathrm{Cl})$ & - & & $0.903(0.877,0$. & & - & & $0.754(0.687,0.826)$ & \\
\hline
\end{tabular}

LoS, length of hospital stay; CDI, Clostridioides (Clostridium) difficile infection; rCDI, recurrent CDI; IQR, interquartile range; s.D., standard deviation; Cl, confidence interval; KM, Kaplan-Meier. ${ }^{\mathrm{a} K M}$ analysis.

${ }^{\text {b}}$ Stratified Cox analysis. 

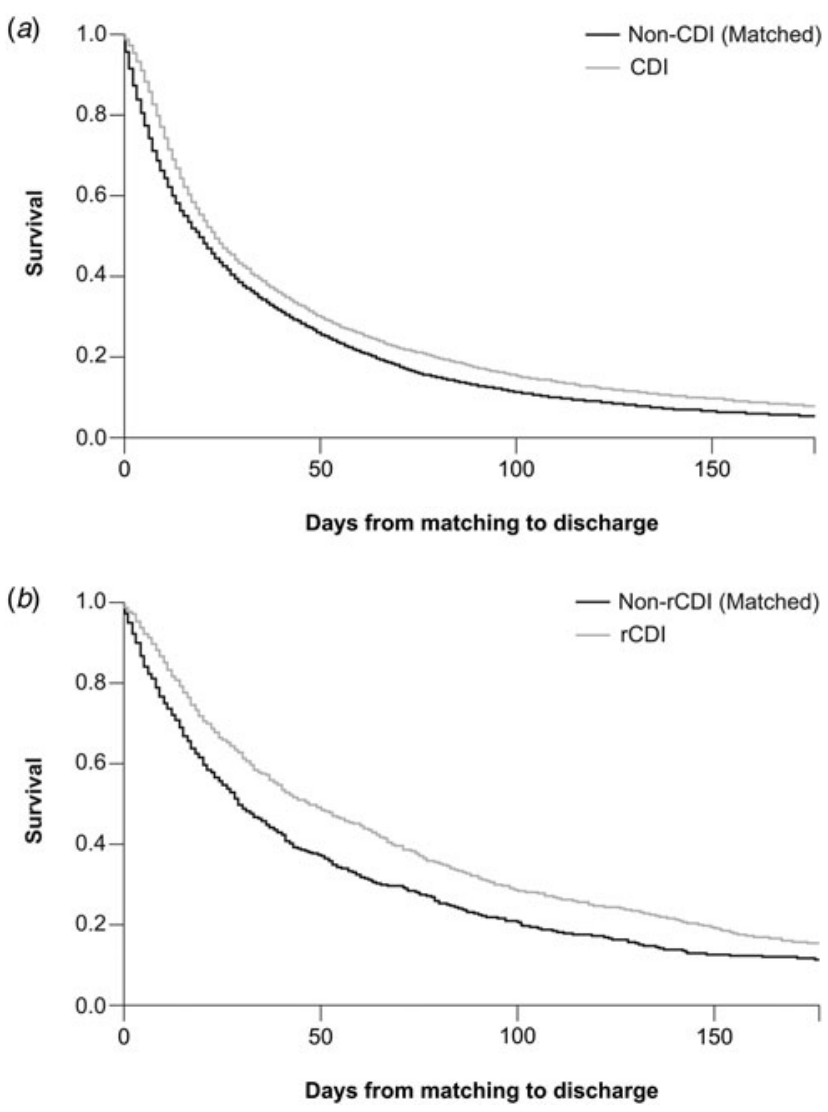

Fig. 2. KM curves with different censoring conditions. (a) Residual LoS in CDI and (b) residual LoS in $\mathrm{rCDI}$. KM, Kaplan-Meier; LoS, length of hospital stay; CDI, Clostridioides (Clostridium) difficile infection; rCDI, recurrent CDI.

and matched non-CDI hospitalisations was 4 days (IQR: 3, 14) (Fig. 2a). The difference in median KM estimates from matching date to discharge between the rCDI cohort and matched non-rCDI cohort was 19 days (IQR: 6, 34) (Fig. 2b). The adjusted hazard ratio for discharge was 0.903 (95\% CI 0.877-0.930) for CDI and 0.754 (95\% CI $0.687-0.826)$ for rCDI.

\section{Excess mortality}

The difference in mortality between non-CDI and CDI hospitalisations was $17.2 \%$ (95\% CI 16.4-17.9) before matching (Table 4). After matching, the CDI-attributable adjusted excess mortality rate was $6.9 \%$ (95\% CI 5.9-7.9), and the adjusted mortality hazard ratio was 1.372 (95\% CI 1.289-1.459).

The difference in mortality between non-rCDI and rCDI hospitalisations was $2.9 \%$ (95\% CI $0.5-5.4)$ before matching, and after matching, the rCDI-attributable mortality was $-1.9 \%(95 \%$ CI $-5.2-1.4)$. The mortality hazard ratio was 0.730 (95\% CI $0.627-0.851)$.

\section{Excess costs}

The median difference in cost between non-CDI and CDI hospitalisations during the entire hospital stay was JPY 1327615 (IQR: $710,917,2,379,678$ ) before matching (Table 5). The median difference in cost between the matched CDI and non-CDI pairs during the residual stay was JPY 130296 (IQR: -417,399, 749,960).
Similarly, the median difference in cost between non-rCDI and rCDI hospitalisation during the entire stay was JPY 1666828 (IQR: $1,219,072,2,685,444)$. The median difference in cost between the matched rCDI and non-rCDI pair during the residual stay was JPY 81054 (-1,064,060, 1,139,728).

\section{Discussion}

This study investigated nearly 6 million hospitalisations during a 9-year study period using a large, Japanese, hospital-based administrative database. A total of 11823 hospitalisations were defined as associated with CDI and 1359 of these were defined as associated with rCDI. Propensity score matching at the time of the CDI/rCDI onset was applied to adjust baseline and time-varying confounding as well as immortal time bias. The median excess LoS attributable to CDI and rCDI was 3 days and 6.5 days, respectively. The excess mortality attributable to CDI was $6.9 \%$, and there was no excess mortality attributable to $\mathrm{rCDI}(-1.9 \%)$.

The studies in various countries have shown that excess LoS was much shorter when immortal time bias was taken into account in the analysis: 12 days (median) vs. 0.9 days (mean) in Australia [6], 21 days (median) vs. 7 days (mean) in the UK [7], 26 days vs. 6 days (median) in Canada [10], 14 days vs. 2.27 days (mean) in a general hospitalised population in the US [8] and 8.4 days vs. 5.1 days in sepsis survivors in the US [9].

Previous studies have also shown that CDI reduces hospital discharge rates. For example, in the UK, CDI reduced the daily discharge rate by about $28 \%$ compared with non-CDI [7], and in Australia and Canada, CDI reduced the daily discharge rate by about $45-60 \%[6,10]$. Our study showed that the difference in median LoS between CDI and non-CDI hospitalisations was 40 days before matching but by adjusting confounding factors and immortal time bias, it was substantially shorter at 3 days. CDI vs. non-CDI reduced the daily discharge rate by $\sim 10 \%$. The relatively large difference in excess LoS before and after the matching, as well as the relatively small effect of CDI on observed daily discharge rates, might be because hospital stays tend to be longer in Japan than in other countries. For example, Organization for Economic Cooperation and Development data for the period 2000-2015 show that the mean LoS for all causes was 7.8 days for 35 member countries overall, whereas for Japan, the mean was 16.5 days - the longest LoS of any country [24].

In previous studies using a multistate model, there was no marked difference in the discharge hazard ratio when hospital death and discharge alive were handled separately, or when death and discharge were combined as a single outcome $[6,8]$. Although hospital death and discharge alive are competing risks, our objective was to estimate the excess LoS as a major cost driver attributable to CDI and, as such, how the hospital stay ended was not critical. However, definition of censoring events largely depends on the study objective, and if CDI burden, including death, is to be considered, this should be taken into account.

There are no estimates of CDI-attributable mortality in Japan that are directly comparable to our study. Other studies have reported an all-cause, 30-day mortality rate of $3.4-15.1 \%$ in patients with CDI $[13,25-30]$. In our study, the all-cause hospital mortality in the CDI hospitalisation rate was $22.1 \%$, which was higher than previously reported from Japan but similar to the hospital mortality in the US study of patients with sepsis 
Table 4. Summary of mortality outcomes in the total CDI and rCDI cohorts and in the matched CDI and rCDI cohorts

\begin{tabular}{|c|c|c|c|c|c|c|c|c|}
\hline & \multicolumn{2}{|c|}{ Before matching } & \multicolumn{2}{|c|}{ After matching } & \multicolumn{2}{|c|}{ Before matching } & \multicolumn{2}{|c|}{ After matching } \\
\hline & Non-CDI & CDI & Non-CDI & CDI & Non-rCDI & rCDI & Non-rCDI & rCDI \\
\hline $\begin{array}{l}N \text { unique } \\
\text { hospitalisation }\end{array}$ & 5982231 & 11823 & 11461 & 11607 & 10464 & 1359 & 1096 & 1320 \\
\hline $\begin{array}{l}N \text { matched } \\
\text { hospitalisations }\end{array}$ & - & - & 11607 & 11607 & - & - & 1320 & 1320 \\
\hline $\begin{array}{l}\text { Hospital death, } \\
n(\%)\end{array}$ & 293001 (4.9) & $2607(22.1)$ & $1697(14.6)$ & $2499(21.5)$ & $2272(21.7)$ & $335(24.7)$ & $345(26.1)$ & $320(24.2)$ \\
\hline $\begin{array}{l}\text { Excess } \\
\text { mortality, \% } \\
(95 \% \mathrm{Cl})\end{array}$ & \multicolumn{2}{|l|}{$17.2(16.4,17.9)^{a}$} & \multicolumn{2}{|l|}{$6.9(5.9,7.9)^{\mathrm{b}}$} & \multicolumn{2}{|l|}{$2.9(0.5,5.4)^{a}$} & \multicolumn{2}{|c|}{$-1.9(-5.2,1.4)^{\mathrm{b}}$} \\
\hline $\begin{array}{l}\text { Mortality } \\
\text { hazard ratio } \\
(95 \% \mathrm{Cl})\end{array}$ & \multicolumn{2}{|l|}{-} & \multicolumn{2}{|c|}{$1.372(1.289,1.459)^{c}$} & \multicolumn{2}{|l|}{-} & \multicolumn{2}{|c|}{$0.730(0.627,0.851)^{c}$} \\
\hline
\end{tabular}

$\mathrm{CDI}$, Clostridioides (Clostridium) difficile infection; rCDI, recurrent $\mathrm{CDI}$; $\mathrm{Cl}$, confidence interval.

${ }^{\mathrm{a}}$ Crude estimate.

${ }^{\mathrm{b}} \mathrm{P}<0.001$ by McNemar test.

${ }^{\mathrm{c}}$ Stratified Cox analysis.

(24.0\%). It has to be noted, however, our CDI cohort was much older than the patients in the US study (81 years compared with 70.9 years) [9].

A major factor in managing nosocomial CDI is avoiding relapse or re-infection, particularly among patients with risk factors $[12,25,31,32]$. In a recent systematic review of studies in Japan, the rate of rCDI was 3.3-27.3\% [12], and in a matchedcohort study of rCDI using the same Japanese hospital database as our study, the rate of rCDI was estimated at $18 \%$ [31]. In our study, before matching, the total LoS in rCDI hospitalisations was 62 days; after matching, the adjusted rCDI-attributable median residual LoS was 6.5 days, yet with censoring transfer to another hospital, it was 19 days. rCDI had a relatively large effect on hospital discharge, reducing the daily discharge rate by $24.6 \%$. Of note, in unmatched rCDI and non-rCDI hospitalisations, the difference in mortality was $2.9 \%$, yet after matching, it was similar between the groups, such that there was no excess mortality $(-1.9 \%)$. This differs from previous findings in Europe [33] and the United States [34] where rCDI was independently associated with excess mortality. This difference in mortality risk may reflect the lower incidence of CDI strains associated with severe infection (e.g. ribotype 027) in Japan vs. other regions in the world [12, 35]. Also, hospitalised patients who were critically ill may have been less likely to have received a diagnosis code or been treated for CDI.

In our analysis, the median excess cost during the residual stay was JPY 130296 (USD 1185). In the only previous large-scale study of CDI in a hospitalised general population in Japan, the CDI-attributable excess cost was USD 3213 [4]. The difference can be explained by the use of different target populations, i.e. DPC hospitals only in our analysis compared with all hospitals in Fukuda et al., and different durations for cost estimation, i.e. only after CDI onset as opposed to entire stay.

The main limitation of the study was the inclusion of patient records only from DPC hospitals, where the reimbursement limit is determined by LoS and which may have different treatment policies to non-DPC hospitals. For example, overall LoS is known to be shorter in DPC hospitals [36]. Even if CDI and non-CDI hospitalisations were managed under the same policy, the excess LoS may be shorter or longer in non-DPC hospitals. In addition, we are aware the treatment pattern for CDI changed over the time period of this study [37] so it is not known if the excess LOS for the entire period can be generalised to an overall population of contemporary Japanese inpatients. Moreover, Japan has a unique 'super-ageing' society [38] and long LoS compared to other countries [24], and universal insurance coverage may have affected treatment policy. As such, the results from this study may not be generalisable to other parts of the world. However, we consider it important to measure the impact of CDI using appropriate methodology in different medical settings.

Other limitations were that mortality was assessed as hospital death and that death after discharge was not captured. Also, CDI may have been over- or under-represented as clinical symptoms were not captured and EIA test results were limited. However, we believe underestimation was more likely for several reasons: (1) only around half of cases treated with oral VCM or oral/IV MNZ had a diagnosis code (i.e. low sensitivity of diagnosis code-based definition) [36] and (2) EIA test results were available from only nine hospitals and the majority of CDI was defined by diagnosis and CDI treatment, while acknowledging that not all CDI cases in Japan are managed with VCM or MNZ (e.g. some cases are managed by stopping antibiotic treatment) [31]. Any misclassified false-negative cases would bias towards a smaller difference between CDI and non-CDI (i.e. an excess LoS that might have been larger than that reported). Although, owing to the limited proportion of CDI cases among the total cohort, there would be little impact on primary outcome. Additionally, rCDI episodes included in our analysis were only those that occurred during the index hospitalisation (i.e. rCDI episodes occurring after discharge were not included); nevertheless, the rCDI rate $(11.5 \%)$ in our analysis was in the range reported previously in Japan [12].

\section{Conclusion}

In this study, propensity score matching at the time of the CDI/ rCDI onset was applied to adjust baseline and time-varying 
Table 5. Summary of costs in the total CDI/rCDI cohorts and the matched CDI/rCDI cohorts

\begin{tabular}{|c|c|c|c|c|c|c|c|c|}
\hline & \multicolumn{2}{|c|}{ Before matching } & \multicolumn{2}{|c|}{ After matching } & \multicolumn{2}{|c|}{ Before matching } & \multicolumn{2}{|c|}{ After matching } \\
\hline & Non-CDI & CDI & Non-CDI & CDI & Non-rCDI & rCDI & Non-rCDI & rCDI \\
\hline $\begin{array}{l}\text { Hospitalisation, } \\
n\end{array}$ & 5982231 & 11823 & 11461 & 11607 & 10464 & 1359 & 1096 & 1320 \\
\hline \multicolumn{9}{|c|}{ Medical expenditure (JPY) } \\
\hline Median (IQR) & $\begin{array}{c}634967 \\
(370,930 \\
1,196,608)\end{array}$ & $\begin{array}{c}1962582 \\
(1,081,847 \\
3,576,287)\end{array}$ & $\begin{array}{c}1891891 \\
(1,052,184, \\
3,344,203)\end{array}$ & $\begin{array}{c}1941227 \\
(1,076,655, \\
3,484,586)\end{array}$ & $\begin{array}{c}1808902 \\
(1,010,982, \\
3,266,417)\end{array}$ & $\begin{array}{c}3475730 \\
(2,230,054, \\
5,951,861)\end{array}$ & $\begin{array}{c}3210105 \\
(2,014,354, \\
5,367,351)\end{array}$ & $\begin{array}{c}3426872 \\
(2,192,061, \\
5,785,769)\end{array}$ \\
\hline Mean (s.D.) & $\begin{array}{c}990426 \\
(1247496)\end{array}$ & $\begin{array}{c}3170660 \\
(4142315)\end{array}$ & $\begin{array}{c}2745418 \\
(3064840)\end{array}$ & $\begin{array}{c}2993468 \\
(3544578)\end{array}$ & $\begin{array}{c}2912329 \\
\text { (3 } 888042)\end{array}$ & $\begin{array}{c}5159751 \\
(5331983)\end{array}$ & $\begin{array}{c}4788687 \\
(5275456)\end{array}$ & $\begin{array}{c}5104966 \\
(5347836)\end{array}$ \\
\hline \multirow[t]{2}{*}{ Range } & 0-836 403985 & $7040-91703212$ & $0-84164129$ & $7040-64651534$ & $7040-91703212$ & $369918-69105695$ & $248603-67463837$ & $369918-69105695$ \\
\hline & \multicolumn{2}{|l|}{ During entire stay } & \multicolumn{2}{|c|}{ During residual stay } & \multicolumn{2}{|l|}{ During entire stay } & \multicolumn{2}{|l|}{ During residual stay } \\
\hline \multicolumn{9}{|c|}{ Excess medical expenditure (JPY) } \\
\hline Median (IQR) & \multicolumn{2}{|c|}{$1327615(710,917,2,379,678)$} & \multicolumn{2}{|c|}{$130296(-417,399,749,960)$} & \multicolumn{2}{|c|}{$1666828(1,219,072,2,685,444)$} & \multicolumn{2}{|c|}{$81054(-1,064,060,1,139,728)$} \\
\hline Mean $(95 \% \mathrm{Cl})$ & \multicolumn{2}{|c|}{$2180234(2,105,552,2,254,915)$} & \multicolumn{2}{|c|}{$232163(175,838,288,487)$} & \multicolumn{2}{|c|}{$2247423(1,954,086,2,540,760)$} & \multicolumn{2}{|c|}{$-54484(-298,220,189,252)$} \\
\hline \multicolumn{9}{|c|}{ Excess medical expenditure (USD) } \\
\hline Median (IQR) & \multicolumn{2}{|c|}{$12069(6,463,21,633)$} & \multicolumn{2}{|c|}{$1185(-3,795,6818)$} & \multicolumn{2}{|c|}{$15153(11,082,24,413)$} & \multicolumn{2}{|l|}{$737(-9,673,10,361)$} \\
\hline Mean $(95 \% \mathrm{Cl})$ & \multicolumn{2}{|c|}{$19820(19,141,20,499)$} & \multicolumn{2}{|c|}{$2111(1,599,2623)$} & \multicolumn{2}{|c|}{$20431(17,764,23,098)$} & \multicolumn{2}{|l|}{$-495(-2,711,1720)$} \\
\hline
\end{tabular}

CDI, Clostridioides (Clostridium) difficile infection; rCDI, recurrent CDI; JYP, Japanese Yen; IQR, interquartile range; CI, confidence interval; USD, United States Dollar (conversion rate USD 1= JPY 110). 
confounding as well as immortal time bias. Our data describe the burden of CDI and rCDI on LoS, mortality and costs, and should support a fair decision making and resource allocation for CDI management in Japanese hospital settings.

Supplementary material. The supplementary material for this article can be found at https://doi.org/10.1017/S0950268820000606.

Data. Researchers may request access to anonymised participant level data, trial level data and protocols from Astellas sponsored clinical trials at www. clinicalstudydatarequest.com.

Acknowledgements. This study was initiated and supported by Astellas Pharma, Inc. Medical writing support was provided by Annick Moon, PhD, of Cello Health MedErgy and funded by Astellas Pharma, Inc.

Author contributions. All authors contributed to study design, analysis and review of the manuscript.

Financial support. This study was initiated and funded by Astellas Pharma, Inc. Medical writing support was funded by Astellas Pharma, Inc.

Conflict of interest. TK and TS are employees of Astellas Pharma, Inc. SS is an employee of Astellas Pharma US LLC.

\section{References}

1. Debast SB, Bauer MP and Kuijper EJ (2014) European Society of Clinical Microbiology and Infectious Diseases: update of the treatment guidance document for Clostridium difficile infection. Clinical Microbiology and Infection 20(suppl. 2), 1-26.

2. Kunishima $\mathbf{H}$ et al. (2018) Healthcare burden of recurrent Clostridioides difficile infection in Japan: a retrospective database study. Journal of Infection and Chemotherapy 24, 892-901.

3. McDonald LC et al. (2018) Clinical practice guidelines for Clostridium difficile infection in adults and children: 2017 update by the Infectious Diseases Society of America (IDSA) and Society for Healthcare Epidemiology of America (SHEA). Clinical Infectious Diseases 31, 431-455.

4. Fukuda H, Yano T and Shimono N (2018) Inpatient expenditures attributable to hospital-onset Clostridium difficile infection: a nationwide casecontrol study in Japan. PharmacoEconomics 36, 1367-1376.

5. Magee G et al. (2015) Impact of Clostridium difficile-associated diarrhea on acute care length of stay, hospital costs, and readmission: a multicenter retrospective study of inpatients, 2009-2011. American Journal of Infection Control 43, 1148-1153.

6. Mitchell BG et al. (2014) The prolongation of length of stay because of Clostridium difficile infection. American Journal of Infection Control 42, 164-167.

7. van Kleef E et al. (2014) Excess length of stay and mortality due to Clostridium difficile infection: a multi-state modelling approach. The Journal of Hospital Infection 88, 213-217.

8. Stevens VW et al. (2015) Excess length of stay attributable to Clostridium difficile infection (CDI) in the acute care setting: a multistate model. Infection Control and Hospital Epidemiology 36, 1024-1030.

9. Lagu T et al. (2014) The impact of hospital-onset Clostridium difficile infection on outcomes of hospitalized patients with sepsis. Journal of Hospital Medicine 9, 411-417.

10. Forster AJ et al. (2012) The effect of hospital-acquired infection with Clostridium difficile on length of stay in hospital. Canadian Medical Association Journal 184, 37-42.

11. Kunishima H (2008) The Japanese clinical practice guidelines for management of Clostridioides (Clostridium) difficile infections. Tokyo: Japanese Society of Chemotherapy, The Japanese Association for Infectious Diseases. Published online: 2008.

12. Riley TV and Kimura T (2018) The epidemiology of Clostridium difficile infection in Japan: a systematic review. Infectious Diseases and Therapy 7, 39-70.
13. Yasunaga $\mathbf{H}$ et al. (2012) The burden of Clostridium difficile-associated disease following digestive tract surgery in Japan. The Journal of Hospital Infection 82, 175-180.

14. Mitchell BG and Gardner A (2012) Prolongation of length of stay and Clostridium difficile infection: a review of the methods used to examine length of stay due to healthcare associated infections. Antimicrobial Resistance and Infection Control 1: 14.

15. Beyersmann J et al. (2009) Nosocomial infection, length of stay, and timedependent bias. Infection Control and Hospital Epidemiology 30, 273-276.

16. Beyersmann J et al. (2008) An easy mathematical proof showed that timedependent bias inevitably leads to biased effect estimation. Journal of Clinical Epidemiology 61, 1216-1221.

17. Shintani AK et al. (2009) Immortal time bias in critical care research: application of time-varying Cox regression for observational cohort studies. Critical Care Medicine 37, 2939-2945.

18. Zhou $\mathrm{Z}$ et al. (2005) Survival bias associated with time-to-treatment initiation in drug effectiveness evaluation: a comparison of methods. American Journal of Epidemiology 162, 1016-1023.

19. Japanese Society for Pharmacoepidemiology. Databases available for pharmacoepidemiology researches in Japan (information obtained from survey answers as of October 2018). Available at http://www.jspe.jp/mtstatic/FileUpload/files/JSPE_DB_TF_E.PDF (Accessed 14 June 2019).

20. Public Policy Committee, International Society of Pharmacoepidemiology (2016) Guidelines for good pharmacoepidemiology practice (GPP). Pharmacoepidemiology and Drug Safety 25, 2-10.

21. Quan H et al. (2005) Coding algorithms for defining comorbidities in ICD-9-CM and ICD-10 administrative data. Medical Care 43, 1130-1139.

22. Kimura T et al. (2020) Validation and recalibration of Charlson and elixhauser comorbidity indices to predict in-hospital mortality in hospitalized patients in a Japanese hospital-based administrative database. Japanese Journal of Pharmacoepidemiology 25, 1-14.

23. Austin PC (2011) Optimal caliper widths for propensity-score matching when estimating differences in means and differences in proportions in observational studies. Pharmaceutical Statistics 10, 150-161.

24. OECD (2017) Health at a Glance 2017: OECD Indicators. Paris: OECD Publishing. Available at http://dx.doi.org/10.1787/health_glance-2017-en (Accessed 14 June 2019).

25. Honda $\mathbf{H}$ et al. (2014) Incidence and mortality associated with Clostridium difficile infection at a Japanese tertiary care center. Anaerobe 25, 5-10.

26. Matsumoto K et al. (2014) Factors affecting treatment and recurrence of Clostridium difficile infections. Biological \& Pharmaceutical Bulletin 37, 1811-1815.

27. Hikone $\mathbf{M}$ et al. (2015) Risk factors for recurrent hospital-acquired Clostridium difficile infection in a Japanese university hospital. Clinical and Experimental Gastroenterology 8, 191-196.

28. Kobayashi K et al. (2017) Adherence to clinical practice guidelines for the management of Clostridium difficile infection in Japan: a multicenter retrospective study. European Journal Of Clinical Microbiology \& Infectious Diseases 36, 1947-1953.

29. Mori $\mathbf{N}$ et al. (2015) Incorrect diagnosis of Clostridium difficile infection in a university hospital in Japan. Journal of Infection and Chemotherapy 21, 718-722.

30. Takahashi M, Mori N and Bito S (2014) Multi-institution case-control and cohort study of risk factors for the development and mortality of Clostridium difficile infections in Japan. BMJ Open 4, e005665.

31. Kimura T, Snijder R and Sugitani T (2019) Characterization and risk factors for recurrence of Clostridium difficile infection in Japan: a nationwide real-world analysis using a large hospital-based administrative dataset. Journal of Infection and Chemotherapy 25, 615-620.

32. Zilberberg MD et al. (2017) Recurrent Clostridium difficile infection among Medicare patients in nursing homes: a population-based cohort study. Medicine 96, e6231.

33. Kuntz JL et al. (2017) Utilization of health services among adults with recurrent Clostridium difficile infection: a 12-year population-based study. Infection Control and Hospital Epidemiology 38, 45-52.

34. Olsen MA et al. (2015) Recurrent Clostridium difficile infection is associated with increased mortality. Clinical Microbiology and Infection 21, 164-170. 
35. Freeman J et al. (2010) The changing epidemiology of Clostridium difficile infections. Clinical Microbiology Reviews 23, 529-549.

36. Ministry of Health, Labour and Welfare. Investigation of patient discharge following 2016 introduction of DPC. Available at https:// www.mhlw.go.jp/stf/shingi2/0000196043.html (Accessed 14 June 2019).
37. Kimura T et al. (2020) Clostridioides (Clostridium) difficile infection in Japanese hospitals 2008-2017: a real-world nationwide analysis of treatment pattern, incidence and testing density. Journal of Infection and Chemotherapy. In press.

38. McCurry J (2015) Japan will be model for future super-ageing societies. Lancet (London, England) 386, P1523. 